\title{
Intervención antrópica y su impacto sobre la estructura y composición de la vegetación en la cuenca El Caraño de Quibdó, Chocó, Colombia
}

\author{
Human disturbance and its impact on the structure and composition of \\ vegetation at El Caraño basin of Quibdó, Chocó, Colombia
}

\section{Luis A. Mosquera Maturana ${ }^{1}$, Hamleth Valois-Cuesta ${ }^{2}$, Harley Quinto-Mosquera²}

\begin{abstract}
Resumen
El Chocó es una región reconocida por sus altos índices de biodiversidad y variedad de ecosistemas. Lamentablemente, los ecosistemas estratégicos, como las microcuencas urbanas que atraviesan los centros poblados, las cuales experimentan perturbaciones antrópicas que las llevan a un estado de deterioro y las limita en sus posibilidades de ofrecer bienes y servicios ambientales. El presente trabajo caracterizó la flora asociada con la microcuenca El Caraño de la ciudad de Quibdó, Chocó, con la finalidad de generar información que permita apoyar acciones que lleven a valorar, conservar y usar sosteniblemente este ecosistema. Se realizaron muestreos de vegetación en la zona alta, media y baja, se establecieron seis parcelas de $20 \times 4 \mathrm{~m}\left(160 \mathrm{~m}^{2}\right.$ por zona), registrando todas las plantas espermatofitas con diámetro a la altura del pecho (DAP) superior a $1 \mathrm{~cm}$. Se registraron 69 especies, distribuidas en 45 géneros y 30 familias. Las familias más representativas fueron Melastomataceae (11), Arecaceae (9), Rubiaceae (8), Araceae (5), Fabaceae (3) y Piperaceae (3). Los valores de diversidad fueron altos (>3) en las tres zonas, determinados por comunidades vegetales con especies raras y poco abundantes, especialmente en la zona media y baja donde el deterioro ambiental es más evidente. Estos resultados muestran que desde el punto de vista de la composición y estructura de la vegetación la zona alta ha sufrido menos impactos y transformaciones que la flora de las zonas media y baja, por lo que, debería ser usada como modelo para la restauración ecológica de la microcuenca en su conjunto.
\end{abstract}

Palabras clave: Deterioro ambiental, El Caraño, Especies indicadoras, Impacto antrópico, Selva chocoana.

\begin{abstract}
Choco is a region renowned for its high levels of biodiversity and variety of ecosystems. Unfortunately, strategic ecosystems as watersheds that cross urban population centers, which suffer human disturbance that lead to a state of deterioration and limited in their ability to provide environmental goods and services. This study characterized the flora associated with the watershed the city Caraño Pretoria, Chocó, in order to generate information to support actions that lead to value, conserve and sustainably use ecosystem. Vegetation sampling were conducted in the high, medium and low, were established six plots of $20 \times 4 \mathrm{~m}$ (160 $\mathrm{m}^{2}$ per zone), recording all flowering plants with a diameter at breast height $(D B H)$ greater than $1 \mathrm{~cm}$. We recorded 69 species representing 45 genera and 30 families. Most representative families were Melastomataceae (11), Arecaceae (9), Rubiaceae (8), Araceae (5), Fabaceae (3) and Piperaceae (3). Diversity values were high $(>3)$ in the three areas,
\end{abstract}

1. Instituto de Investigaciones Ambientales del Pacífico (IIAP), Quibdó, Chocó, Colombia.

2. Programa de Biología, Facultad de Ciencias Básicas, Universidad Tecnológica del Chocó, Quibdó, Colombia.

e-mail: hamleth.valois@alumnos.uva.es

Recibido: 15 de enero de 2012

Aceptado: 23 de abril de 2012 


\section{Bioetnia Volumen 9 № 2 (julio-diciembre), 2012}

certain plant communities and rare species very abundant, especially in the middle and lower where environmental degradation is more evident. These results show that from the point of view of the composition and structure of the upper vegetation has suffered less impacts and transformations that the flora of the middle and lower areas so should be used as a model for ecological restoration the watershed as a whole.

\section{Keywords: Chocoan forest, El Caraño, Environmental degradation, Human impacts, Indicator species.}

\section{Introducción}

Los bosques neotropicales lluviosos poseen alta diversidad biológica, regulan los ciclos del agua y el carbono a nivel global y poseen alta biomasa al compararse con otros ecosistemas continentales (Sarmiento et al., 2005). En este contexto, los bosques pertenecientes a la región pacífica de Colombia son reconocidos, no solo por sus altos niveles de pluviosidad, $>13,000 \mathrm{~mm}$ anuales en algunos puntos de la región (Poveda-M et al., 2004), sino también por su alta diversidad y endemismo de especies vegetales (Rangel-Ch. y Rivera-Díaz, 2004). En la actualidad, esta región cuanta con registros de 4525 especies de espermatofitas (Rangel-Ch y Rivera-Díaz, 2004), sin embargo, el número estimado se encuentra entre 7000 y 8000 especies (Forero y Gentry 1989). Son muchos los grupos taxonómicos de plantas (170 familias) que pueden encontrarse coexistiendo en este territorio, sin embargo, son altamente predominantes familias como Rubiaceae (342 especies y 72 géneros), Orchideaceae $(250,94)$, Melastomataceae $(225,27)$, Piperaceae $(180,4)$, Araceae $(169,17)$, Asteraceae $(125,72)$, Bromeliaceae (119, 13), Fabaceae $(114,40)$, Clusiaceae $(114,15)$, Gesneriaceae $(108,18)$, Poaceae $(103,52)$, Euphorbiaceae $(100,38)$, Ericaceae $(90,11)$, Arecaceae $(82,30)$ y Cyperaceae $(82$, 20 ), representando el $49 \%$ de las especies y el $43 \%$ de los géneros de la flora regional (Rangel-Ch y Rivera-Díaz, 2004).

Aunque las anteriores características han fomentado el reconocimiento de la región chocoana como un centro de importancia a nivel global en términos de conservación de vida silvestre, en la actualidad muchas comunidades vegetales y ecosistemas estratégicos de la región han sido sometidos a fuerte y constantes perturbaciones antrópicas (crecimiento urbano y problemas ambientales derivados) que han ocasionado modificaciones importantes en términos de la composición, estructura y dinámica ecológica de tales ecosistemas; tal es el caso de la microcuenca El Caraño, una potencial fuente de suministro de agua y recursos de subsistencia para los habitantes de la ciudad de Quibdó, que desde hace algunos años viene siendo sometida a fuertes perturba- ciones generadas por el vertimiento de desechos domésticos a sus aguas y la pérdida de cobertura vegetal en su rivera para la construcción de viviendas.

Por lo anterior, el objetivo principal del presente estudio fue caracterizar las comunidades vegetales presentes en la microcuenca El Caraño, como base para la implementación de herramientas que permitan la toma de decisiones para su gestión ambiental. Para ello, se determinó la riqueza, diversidad y composición de la flora para posteriormente evaluar el estado de conservación biológico en este ecosistema estratégico que atraviesa la ciudad de Quibdó.

\section{Metodología}

Área de estudio. El trabajo de campo se desarrolló durante el año 2012 a lo largo de la microcuenca El Caraño en la ciudad de Quibdó, departamento del Chocó, Colombia. Para la realización de los muestreos la microcuenca se dividió en tres zonas o sectores que se describen a continuación:

Zona alta. Se encuentra ubicada desde el nacimiento de la quebrada en el Alto del Granadillo, hasta los centros recreacionales Río mar y Fuego verde, a una altura de 65 $\mathrm{msnm}$. Esta zona se caracteriza por un relieve de pequeñas terrazas con densos parches de vegetación secundaria en estado avanzado de sucesión donde se pueden apreciar claramente tres estratos de vegetación y un predominio de árboles remanentes como Piptocoma discolor, Cecropia peltata y Brosimun utile, además de algunas especies arbustivas como Guatteria cargadero, Psychotria poepigiana, Siparuna guianensi y Miconia spp. y hierbas emergentes como Hedychium coronarium, Lantana camara, Glossoloma panamense y Hiptis sp. (Figuras 1A-B).

Zona media. Esta se encuentra ubicada desde los estadero Río mar y Fuego verde, hasta el puente del barrio Huapango ubicado sobre la Carrera Sexta, a una altura de $47 \mathrm{msnm}$. Esta zona se caracteriza por tener un relieve de terrenos ondulados, encontrarse altamente urbanizada donde la vegetación ha sufrido un alto impacto antrópico producto de la extracción de elementos arbóreos, predominando así hierbas y arbustos diseminados en algunos parches densos. Entre las especies más visibles se encuentran hierbas como Conobea scoparioides, Hedychium coronarium, Mimosa pudica, Achyranthes sp. y Xanthosoma sp.; arbustos como Siparuna guianensis, Ficus sp. y algunos árboles como Cecropia peltata, Piptocoma discolor, Vochysia sp. Además de los elementos que representan la vegetación nativa, en esta zona por su dinámica antrópica se pueden observar otros componentes de vegetación cultivada como Colocasia esculenta e Inga edulis (Figura 1C).

Zona baja. Se ubica a una altura de $43 \mathrm{msnm}$ y comprende desde el puente ElCaraño sobre la carretera Quibdó-Medellín, 

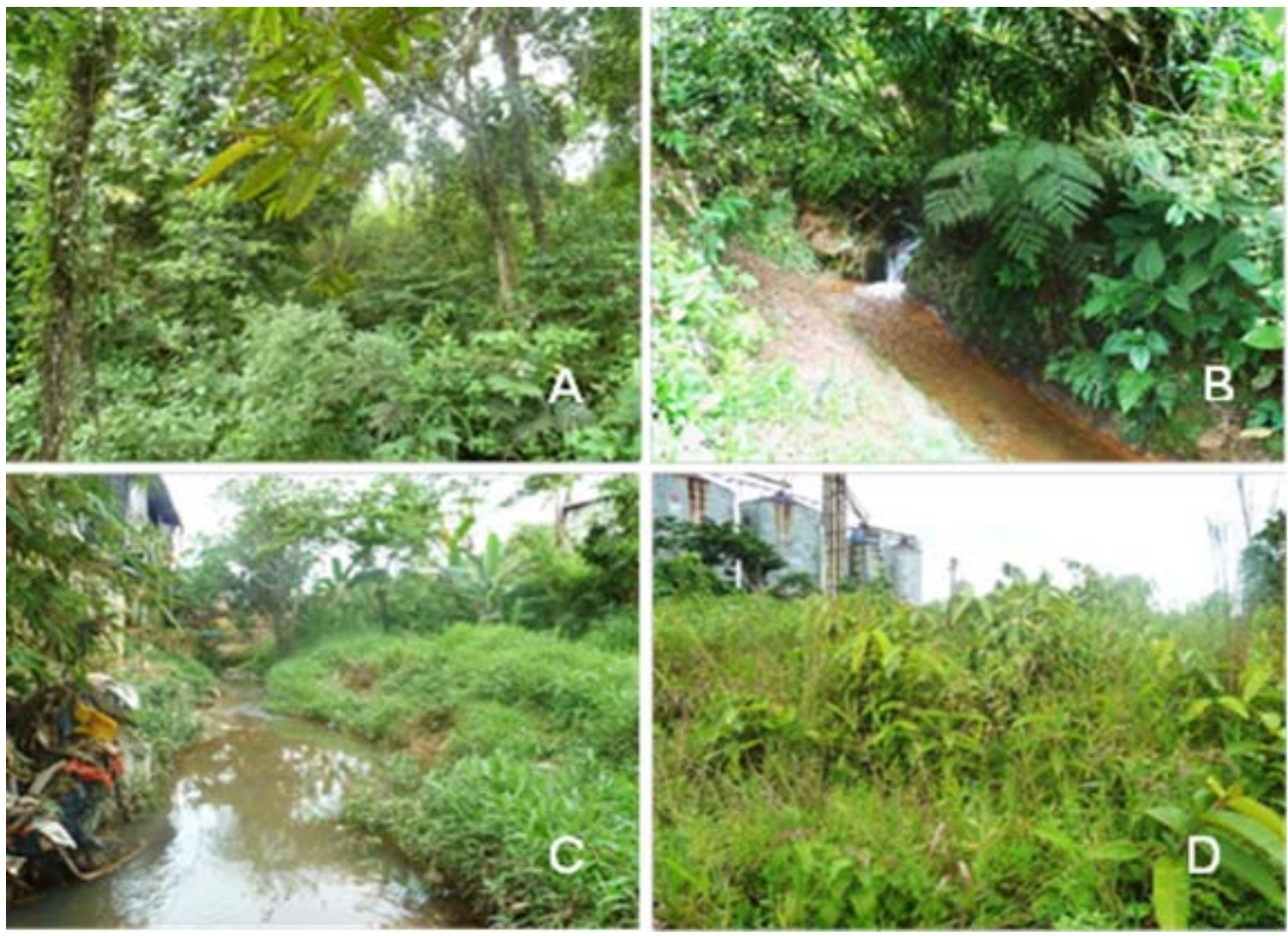

Figura 1. Vegetación predominante en la zona alta (A-B), media (C) y baja (D) de la microcuenca El Caraño de Quibdó, Chocó, Colombia

hasta su desembocadura (en el río Atrato) en el sitio denominado Boca del Caraño en el barrio Kennedy de Quibdó. Se caracteriza por tener un relieve por terrenos inundables con un predominio poblacional de especies herbáceas y otros elementos de vegetación rivereña en bajas densidades. Algunas de las especies que se encuentran en esta zona son Paspalum repens, Cecropia peltata y Pachira acuatica. Se encuentran algunos grupos aislados de especies como Vochysia sp. En términos generales, en esta área se observó una vegetación típica de humedales con una alta densidad de elementos herbáceos, especialmente de la familia Poaceae (Figura 1D).

\section{Métodos}

Levantamientos de vegetación. En cada una de las zonas (alta, media y baja) definidas en la microcuenca se establecieron de manera sistemática (escogiendo áreas con vegetación en casos donde existían centros poblados) dos parcelas de $20 \times 4 \mathrm{~m}\left(160 \mathrm{~m}^{2} \mathrm{o} 0.016\right.$ ha por zona $)$ que comprendieron en total un área de $480 \mathrm{~m}^{2}$ o 0.048 ha. Dentro de cada una de las parcelas se registraron todas las plantas leñosas con diámetro a la altura del pecho (DAP) superior a $1 \mathrm{~cm}$; adicionalmente, se consideraron las plantas superiores no leñosas. A cada uno de los individuos encontrados se le registró el nombre común, nombre científico, género, familia y hábito de crecimiento (hierbas, arbustos y árboles), haciendo recolecta de aquellos individuos preferiblemente en estado fértil. Además, se consideró la percepción de los habitantes del área de influencia sobre algunos aspectos relacionados con los cambios de la cuenca en el tiempo, a través de charlas informales.

Identificación taxonómica de las colecciones. El material recolectado fue identificación taxonómicamente a nivel de familia, género y especie con la ayuda de claves taxonómicas (Gentry 1996) y por medio de confrontación con ejemplares depositados en el Herbario CHOCO y/o descritos en bases de datos como TROPICOS del Missouri Botanical Garden (http://www.tropicos.org) y Herbario COL en línea (http://www.biovirtual.unal.edu.co). Adicional a la identificación taxonómica, los nombre científicos de las especies se corroboraron en las siguientes bases de datos especializadas: The International Plant Names Index (http:/ /www.ipni.org) y The plant list (http://www.theplantlist.org) 
Análisis de los datos. Para evaluar ecológicamente la vegetación asociada con la cuenca El Caraño (en cada zona), se cuantificó el número de especies y se estimó la riqueza esperada de las mismas por medio de curvas de refracción. Adicionalmente, se calculó la diversidad con el índice Margalef, la abundancia con el índice de Simpson y la equidad por medio del índice de Shannon-Wiener (Villareal et al., 2006). Se realizó un análisis de diversidad beta para evaluar el recambio de especies entre las diferentes zonas definidas objeto de estudio (alta, media y baja) en la microcuenca El Caraño. El valor de importancia de las especies dentro de cada zona y entre zonas fue evaluado usando sus abundancias relativas y las diferencias en abundancias relativas fueron comparadas a través de la prueba Chi-cuadrado. Todos los análisis de vegetación se realizaron usando el paquete Vegan del programa R versión 2.15.1 (R Core Team 2012).

Con el fin de determinar el grado de amenaza de las especies encontradas en la microcuenca El Caraño, se revisaron los listados de especies en peligro de la (UICN), donde se les definen dentro de diferentes categorías de amenaza. Sin embargo, para este estudio solo se considerarán aquellas que se encuentren en Peligro Crítico (CR), En Peligro (EN) y Vulnerable (VU).

\section{Resultados y discusión}

Análisis de conservación a través de grupos indicadores. Se registró un total de 69 especies o morfoespecies distribuidas en 45 géneros y 30 familias, aunque el número de géneros podría ser mayor considerando que $21.7 \%$ de los registros encontrados no pudieron ser identificados al nivel de especie. Las familias mejor representadas por número de especies fueron Melastomataceae (11), Arecaceae (9), Rubiaceae (8), Araceae (5), Fabaceae (3) y Piperaceae (3). Las familias restantes presentaron entre dos y una especie (Anexo 1, Figura 2). Este patrón de riqueza de especies dentro de familias botánicas es evidente a nivel regional donde Melastomataceae, Rubiaceae, Araceae y Piperaceae son altamente ricas en especies (Forero y Gentry, 1989, RangelCh. y Rivera-Díaz, 2004), especialmente en aquellas localidades donde los bosques han estado sometidos a disturbios generados por actividad humana (Mosquera y Mosquera, 2002, Bonilla-Luna et al., 2011).

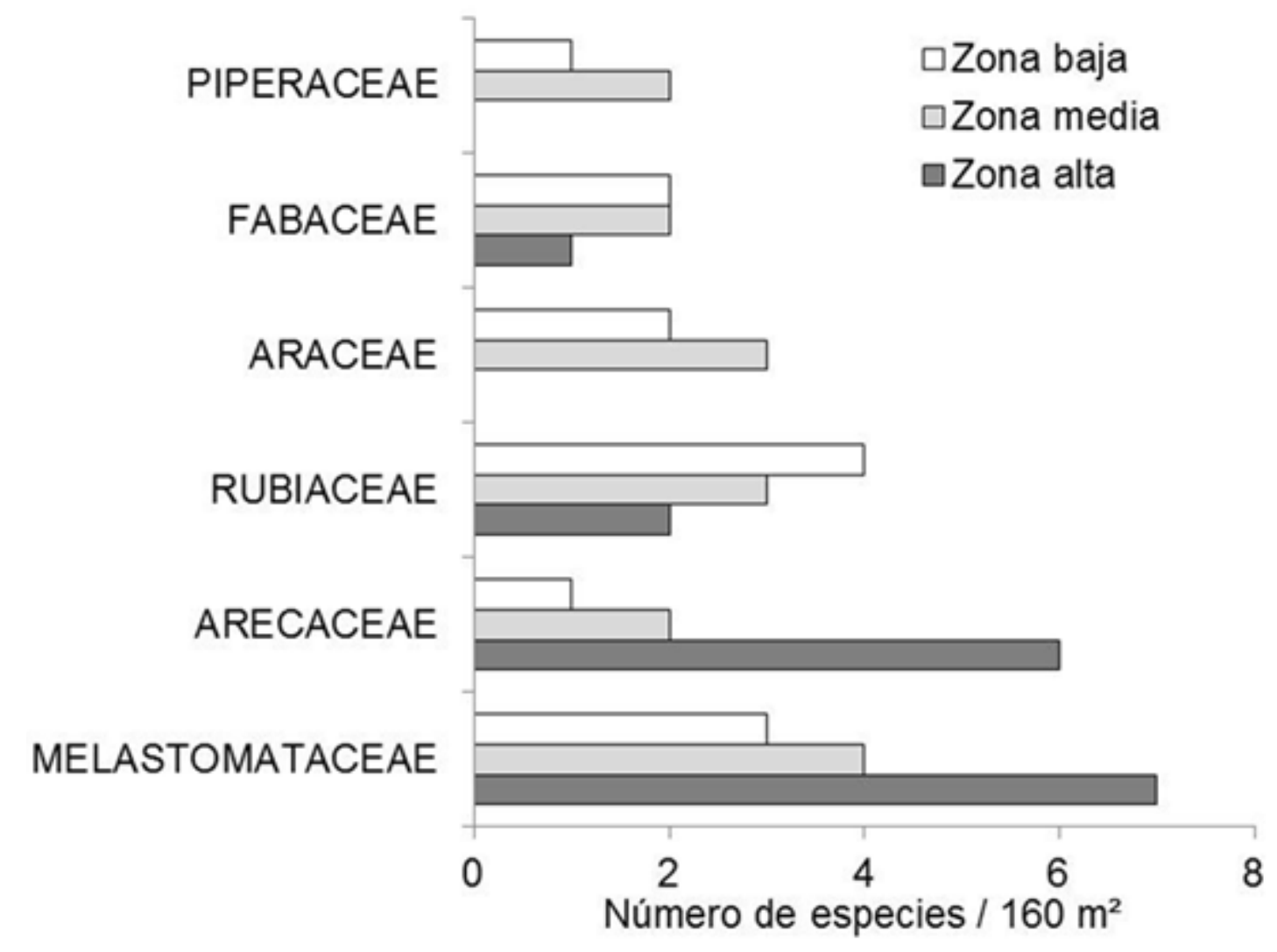

Figura 2. Familias indicadoras y mayormente representativas en término de especies en las zonas alta, media y baja de la microcuenca El Caraño de Quibdó, Chocó 
Intervención antrópica y transformación de la vegetación. LA. Mosquera-Maturana et al.

Al analizar cada una de las zonas estudiadas en la microcuenca El Caraño (alta, media y baja), con base en las familias más representativas, se aprecia una mayor riqueza de especies de las familias Arecaceae y Melastomataceae en la zona alta, mientras que, incluyendo Melastomataceae, Rubiaceae, Araceae, Fabaceae y Piperaceae muestra mayor predominio en las zonas medias y bajas (Figura 3). De hecho, en el Chocó, familias como Piperaceae y Rubiaceae son frecuentemente observadas a orillas de caminos o en llanuras aluviales fuertemente degradas. A excepción de Arecaceae (importante en bosques primarios y/o secundarios en estado avanzado de sucesión y que fue rica en la zona alta), otras familias como Sapotaceae, Chrysobalanaceae y Myristicaceae, propias de bosques maduros en el Chocó (Asprilla et al. 2003), no fueron encontradas en ninguna de las zonas estudiadas, demostrando así que la vegetación asociada con la quebrada El Caraño, en términos generales, ha sido sometida a fuertes presiones producto de las perturbaciones antrópicas que se evidencian especialmente en la parte media y baja de la microcuenca donde la configuración original (al compararlo con la zona alta) de la comunidad vegetal ha sido reemplazada, si se compara con la zona alta, por especies que configuran gremios de plantas oportunistas propias de zonas alteradas. A simple vista, la vegetación de la zona alta presenta un mayor grado de regeneración natural, lo cual se debe a que en las zonas media y baja los disturbios antrópicos se han presentado con mayor intensidad y frecuencia (Figura 1). Por otra parte, aunque en la zona alta no se apreciaron elementos propios de un bosque maduro, el hecho de observar algunos parches de vegetación con remanentes de especies arbóreas como Abarema barbouriana, Piptocoma discolor, Brosimum utile, Oenocarpus bataua, Couratari guianensis y Vochysia sp., pone de manifiesto una configuración más compleja de la estructura de la vegetación en ese sitio de la microcuenca (Figura 1).

Adicional a lo anterior, desde el punto de vista de las especies más representativas a nivel de toda la cuenca, se encontró que especies indicadoras de zonas altamente perturbadas tales como Cecropia peltata, Siparuna guianensis, Vismia macrophylla, Costus villosissimus entre otras del género Piper (Díaz y El Coro 2009), son especialmente predominantes en las zonas media y baja (Figura 3 ). Al hacer

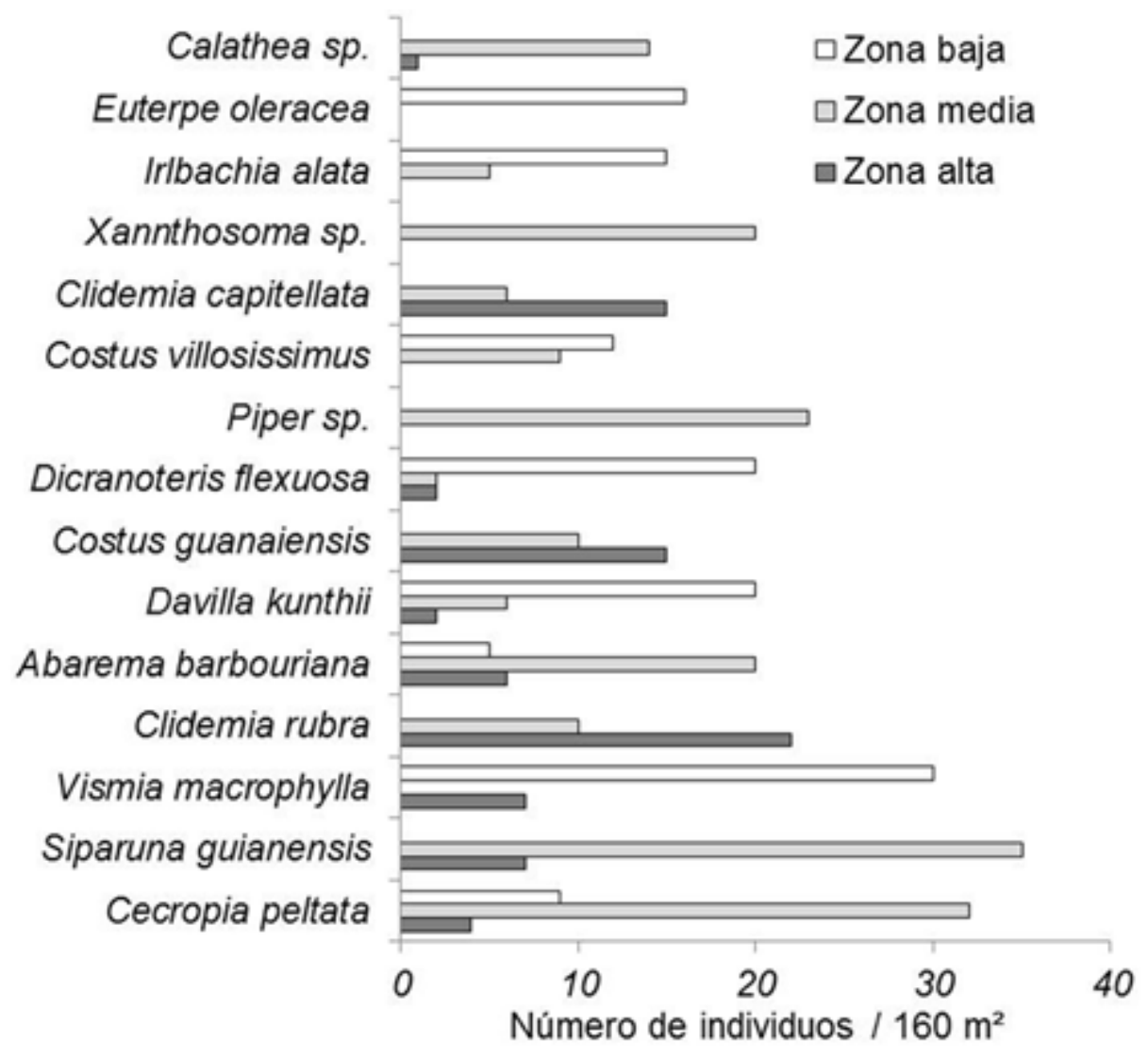

Figura 3. Especies indicadoras y mayormente representativas en término de especies en las zonas alta, media y baja de la microcuenca El Caraño de Quibdó, Chocó 
una comparación con especies abundantes en bosques primarios del Chocó (Palacios et al., 2003), no se apreció evidencia de que las especies registradas aquí como más abundantes, sobre todo en las zonas media y baja (Figura 3) sean importantes desde el punto de vista de sus densidades en ese tipo de bosques, lo cual indica en términos generales, que desde el punto de vista de la composición de la vegetación, la zona alta representa el área con menor degradación y posiblemente lo más parecido a la configuración original de la vegetación en la microcuenca El Caraño.

\section{Análisis de conservación a través de índices ecológicos}

Tanto la riqueza de especies como la abundancia de individuos fueron mayores en las zonas media y alta al compararse con la baja (Tabla 1), sin embargo, es importante resaltar que las curvas de refracción (riqueza vs. abundancias de especies, Figura 5) indicaron que la riqueza de especies observada en las zonas media y baja fue registrada significativamente (las curvas se estabilizan), es decir, que en las zonas media y baja el número de especies podría no incrementar aunque se aumente el esfuerzo de muestreo, caso contrario a lo que ocurrió en la zona alta donde la proyección final de la curva de especies observadas no llega a ser asíntota (no se estabilizó), indicando por consiguiente, que el número de especies en la zona alta puede ser mayor al observado (Figura 5). Lo anterior se debe seguramente a la mayor complejidad estructural (tres estratos que pueden reflejar mayor número de hábitats y grupos funcionales de plantas) que se observa en la zona alta a diferencia de lo que se observa en la zona media y baja donde hay un mayor predominio de especies herbáceas (Figura 1A-B vs. 1C-D).

A pesar del grado de deterioro que experimentan los

Tabla 1

Listado de especies con sus abundancias absolutas registradas en la zona alta, media y baja de la microcuenca El Caraño del municipio de Quibdó, Chocó. Las especies se ordenan desde las más abundantes hasta las más raras

Nombre local

Nombre científico

Familia

Microcuenca El Caraño

Alta Media Baja Total

\begin{tabular}{|c|c|c|c|c|c|c|}
\hline Yarumo & Cecropia peltata L. & Urticaceae & 4 & 32 & 9 & 45 \\
\hline Limón & Siparuna guianensis Aubl. & Siparunaceae & 7 & 35 & & 42 \\
\hline Manchara & Vismia macrophylla Kunth & Hypericaceae & 7 & & 3 & 37 \\
\hline Moritas & Clidemia rubra (Aubl.) Mart. & Melastomataceae & 22 & 1 & & 32 \\
\hline \multirow[t]{2}{*}{ Dormilón } & Abarema barbouriana (Standl.) & & & & & \\
\hline & Barneby \& J.W. Grimes & Fabaceae & 6 & 2 & 5 & 31 \\
\hline Bejuco quemador & Davilla kunthii A.St.-Hil. & Dilleniaceae & 2 & 6 & 2 & 28 \\
\hline Caña agria & Costus guanaiensis Rusby & Costaceae & 15 & 1 & & 25 \\
\hline \multirow[t]{2}{*}{ Helechos } & Dicranopteris flexuosa & & & & & \\
\hline & (Schrad.) Underw. & Gleicheniaceae & 2 & 2 & 2 & 24 \\
\hline NN & Piper sp. & Piperaceae & & 23 & & 23 \\
\hline Caña agria & Costus villosissimus] acq. & Costaceae & & 9 & 12 & 21 \\
\hline Mora blanca & Clidemia capitellat (Bonpl.) D. Don & Melastomataceae & 15 & 6 & & 21 \\
\hline Campana & Chelonanthus alatus (Aubl.) Pulle & Gentianaceae & & 5 & 15 & 20 \\
\hline Palma & Euterpe oleracea Mart. & Arecaceae & & & 16 & 16 \\
\hline Hoja negra & Calathea sp 1 & Marantaceae & 1 & 14 & & 15 \\
\hline Hormigo & Tococa guianensis Aubl. & Melastomataceae & 13 & & & 13 \\
\hline NN & Ernodea littoralisSw. & Rubiaceae & & & 13 & 13 \\
\hline Caucho & Ficus brevibracteata W.C. Burger & Moraceae & & 7 & 5 & 12 \\
\hline NN & Morfo especie 3 & Asteraceae & & 8 & & 8 \\
\hline Licopodiun & Lycopodiella cernua (L.) Pic. Serm. & Licopodiaceae & & 8 & & 8 \\
\hline
\end{tabular}


Intervención antrópica y transformación de la vegetación. LA. Mosquera-Maturana et al.

Tabla 1

Listado de especies con sus abundancias absolutas registradas en la zona alta, media y baja de la microcuenca El Caraño del municipio de Quibdó, Chocó.

Las especies se ordenan desde las más abundantes hasta las más raras (continuación)

\begin{tabular}{|c|c|c|c|c|c|c|}
\hline \multirow[t]{2}{*}{ Nombre local } & \multirow[t]{2}{*}{ Nombre científico } & \multirow[t]{2}{*}{ Familia } & \multicolumn{4}{|c|}{ Microcuenca El Caraño } \\
\hline & & & Alta & Media & Baja & Total \\
\hline Hormigos colorados & Morfo especie 15 & Melastomataceae & 8 & & & 8 \\
\hline Jaboncillo & Isertia pittieri (Standl.) Standl. & Rubiaceae & 4 & 4 & & 8 \\
\hline Sombrito del diablo & Psychotria poeppigiana Standl. & Rubiaceae & & & 8 & 8 \\
\hline Aliso & Piptocoma discolor (Kunth) Pruski & Asteraceae & 4 & 3 & & 7 \\
\hline NN & Morfo especie 4 & Ciclanthaceae & & 7 & & 7 \\
\hline \multirow[t]{2}{*}{ Pacó } & Cespedesia spathulata (Ruiz \& Pav.) & & & & & \\
\hline & Planch & Ochnaceae & 3 & 4 & 4 & 7 \\
\hline NN & Dieffeenbachia sp. & Araceae & & 6 & & 6 \\
\hline Chacarra & Bactris barronis L.H. Bailey & Arecaceae & & 6 & & 6 \\
\hline NN & Morfo especie 5 & Piperaceae & & & 6 & 6 \\
\hline NN & Psychotria sp. & Rubiaceae & & 6 & & 6 \\
\hline NN & Morfo especie 6 & Araceae & & & 5 & 5 \\
\hline Chacarra & Bactris maraja Mart. & Arecaceae & 5 & & & 5 \\
\hline Chacarra & Bactris coloradonis L.H. Bailey & Arecaceae & 5 & & & 5 \\
\hline Paco de monte & Gustavia sp. & Lecythidaceae & 1 & 4 & & 5 \\
\hline Yarumos uva & Pourouma bicolor Mart. & Urticaceae & 4 & & & 4 \\
\hline NN & Morfo especie 7 & Gesneriaceae & & 4 & & 4 \\
\hline NN & Calathea sp. 2 & Maranthaceae & 4 & & & 4 \\
\hline Coronillo & Bellucia pentamera Naudin & Melastomataceae & & 4 & & 4 \\
\hline Flor de mayo & Vochysia sp. & Vochysiaceae & & & 4 & 4 \\
\hline Iraca & Carludovica palmata Ruíz \& Pav. & Cyclanthaceae & & 3 & & 3 \\
\hline Guamo churimo & Inga sp. & Fabaceae & & & 3 & 3 \\
\hline NN & Morfo especie 8 & Orchideaceae & & 3 & & 3 \\
\hline Algodoncillo & Luehea seemannii Triana \& Planch & Malvaceae & & 3 & & 3 \\
\hline Hormigo & Tococa sp. & Melastomataceae & & & 3 & 3 \\
\hline NN & Morfo especie 10 & Piperaceae & & 3 & & 3 \\
\hline NN & Morfo especie 9 & Rubiaceae & & 3 & & 3 \\
\hline NN & Morfo especie 11 & Rubiaceae & & & 3 & 3 \\
\hline NN & Xannthosoma sp. & Araceae & & 2 & & 2 \\
\hline NN & Philodendron sp. & Araceae & & 2 & & 2 \\
\hline NN & Monstera sp. & Araceae & & & 2 & 2 \\
\hline Palma mil peso & Oenocarpus bataua Mart. & Arecaceae & 2 & & & 2 \\
\hline NN & Miconia sp. & Melastomataceae & 2 & & & 2 \\
\hline NN & Siparuna sp. & Siparunaceae & 2 & & & 2 \\
\hline Lechero & Brosimum utile (Hunth) Oken & Moraceae & 2 & & & 2 \\
\hline Cuatro esquina & Morfo especie 1 & Melastomataceae & 1 & & & 1 \\
\hline NN & Morfo especie 2 & Melastomataceae & & & 1 & 1 \\
\hline
\end{tabular}


Bioetnia Volumen 9 № 2 (julio-diciembre), 2012

Tabla 1

Listado de especies con sus abundancias absolutas registradas en la zona alta, media y baja de la microcuenca El Caraño del municipio de Quibdó, Chocó. Las especies se ordenan desde las más abundantes hasta las más raras (continuación)

\begin{tabular}{|c|c|c|c|c|c|c|}
\hline \multirow[t]{2}{*}{ Nombre local } & \multirow[t]{2}{*}{ Nombre científico } & \multirow[t]{2}{*}{ Familia } & \multicolumn{4}{|c|}{ Microcuenca El Caraño } \\
\hline & & & Alta & Media & Baja & Total \\
\hline Cargadero & Anaxagorea crassipetala Hemsl. & Annonaceae & 1 & & & 1 \\
\hline Palma amarga & Welfia regia $\mathrm{H}$. Wendl. & Arecaceae & 1 & & & 1 \\
\hline Taparo & Attalea allenii H.E. Moore & Arecaceae & 1 & & & 1 \\
\hline Palma & $\begin{array}{l}\text { Geonoma cuneata H. Wendl. } \\
\text { ex Spruce }\end{array}$ & Arecaceae & 1 & & & 1 \\
\hline Cabecinegro & Manicaria saccifera Gaerth & Arecaceae & & 1 & & 1 \\
\hline Helecho epifito & Asplenium serratum $\mathrm{L}$. & Aspleniaceae & & & 1 & 1 \\
\hline Guamo bejuco & Inga edulis Mart. & Fabaceae & & 1 & & 1 \\
\hline Guasca pelado & Couratari guianensis Aubl. & Lecythidaceae & 1 & & & 1 \\
\hline Hormigo con capuchón & Tococa sp. & Melastomataceae & 1 & & & 1 \\
\hline NN & Morfo especie 13 & Melastomataceae & & 1 & & 1 \\
\hline NN & Morfo especie 12 & Rubiaceae & 1 & & & 1 \\
\hline Mayo & Palicourea guianensis Aubl. & Rubiaceae & & & 1 & 1 \\
\hline NN & Morfo especie 14 & Solanaceae & 1 & & & 1 \\
\hline NN & $\begin{array}{l}\text { Zamia pirofilla Calonje, D.W. Stev. } \\
\& \text { A. Lindstr. }\end{array}$ & Zamiaceae & 1 & & & 1 \\
\hline Total & & & 150 & 229 & 124 & 584 \\
\hline
\end{tabular}

bosques aledaños a la microcuenca El Caraño, en las tres zonas estudiadas presentan valores de diversidad considerados como importantes según la clasificación de Ramírez (2006). Este autor plantea que comunidades vegetales tropicales donde la diversidad alfa (de Margalef) sea mayor $(>3)$ puede considerarse como muy alta. A pesar de ello, en este aspecto, la zona alta y la zona media no mostraron diferencias entre ellas, pero si con la zona baja, lo cual es evidente al observar el solapamiento de las curvas de refracción entre la zona alta y media, y la diferencia entre estas dos con la curva proyectada para la zona baja. Como los valores de diversidad están determinados por la riqueza de especies y la abundancia de estas dentro de la comunidad vegetal, una explicación a la similitud entre los valores de diversidad de la zona alta y media puede estar dada en que en la zona alta, tal como se comenta antes, la riqueza de especies no fue suficientemente registrada como sí ocurrió en la zona media y baja (Figura 4).

Por otra parte, los valores de diversidad registrados entre la zona alta y la zona media solo indican que en estos dos sectores de la microcuenca existe una similitud numérica en término del número de especies e individuos que albergan esas comunidades vegetales mas no que estas sean parecidas en término de las composiciones de especies o estructura vegetal que las caracteriza; de hecho al analizar el recambio de especies entre zonas a lo largo de la microcuenca, el índice de similitud de Jaccard (diversidad beta) mostró que las tres zonas presentan solo $15 \%$ en sus composiciones florísticas, lo cual está indicando que las zonas difieren altamente entre sí en el tipo de especies que comparten. Lo anterior permite inferir que las composiciones vegetales, en especial en las zonas media y baja, aunque presentan valores de diversidad alfa altos, han sido remplazadas en la gran mayoría de sus elementos originales, lo cual se refleja en aquellas modificaciones en la estructura de estas comunidades, observándose en la parte alta de la microcuenca la presencia de parches de vegetación con predomio de algunos elementos arbóreo mezclados con arbustos y hierbas (en algunos puntos se pueden apreciar tres estratos), caso contrario a lo que se pudo observar en la zona media y baja donde hay una alta presencia de elementos de hábito herbáceo (Figura 1).

Los valores de dominancia y equidad (Tabla 2) mostraron que la estructura de las comunidades vegetales a lo largo de la microcuenca El Caraño está determinada por muchas especies raras y pocas dominantes (Tabla 2), sobre todo en las zonas media y baja (Figura 5). Lo anterior, puede ser explicado al interpretar la curva de rango de abundancias 
Intervención antrópica y transformación de la vegetación. LA. Mosquera-Maturana et al.

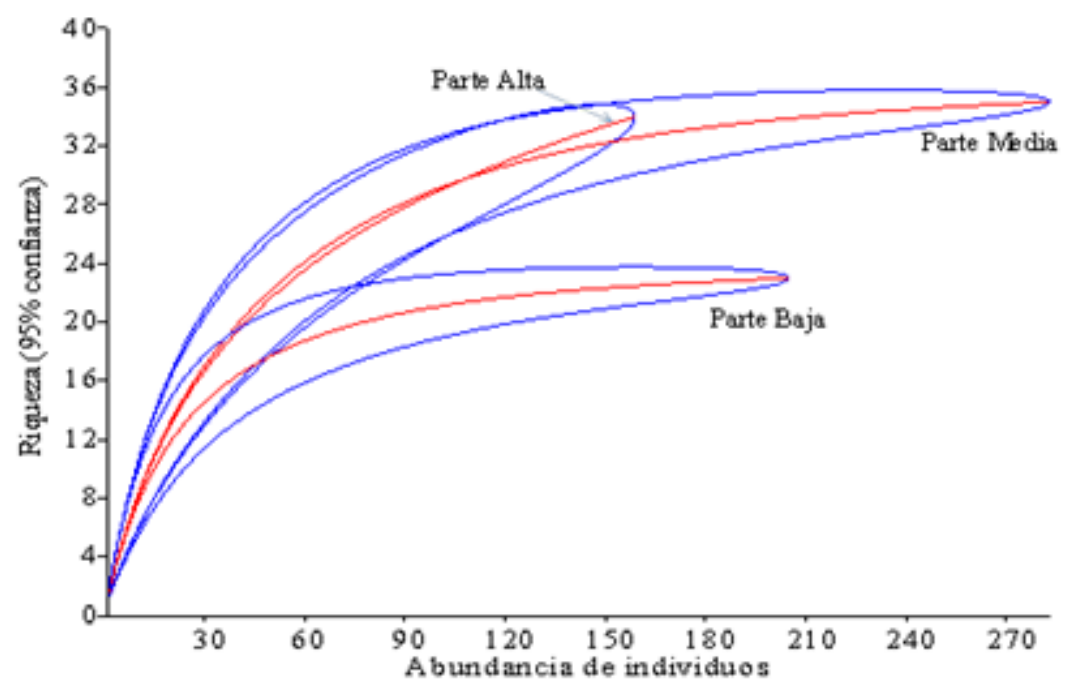

Figura 4. Curva de acumulación de especies (refracción) en la zona alta, media y baja de la microcuenca de El Caraño, Quibdó, Chocó. Las líneas rojas representan la riqueza de especies observada y las líneas azules representan los intervalos de confianza al 95\%

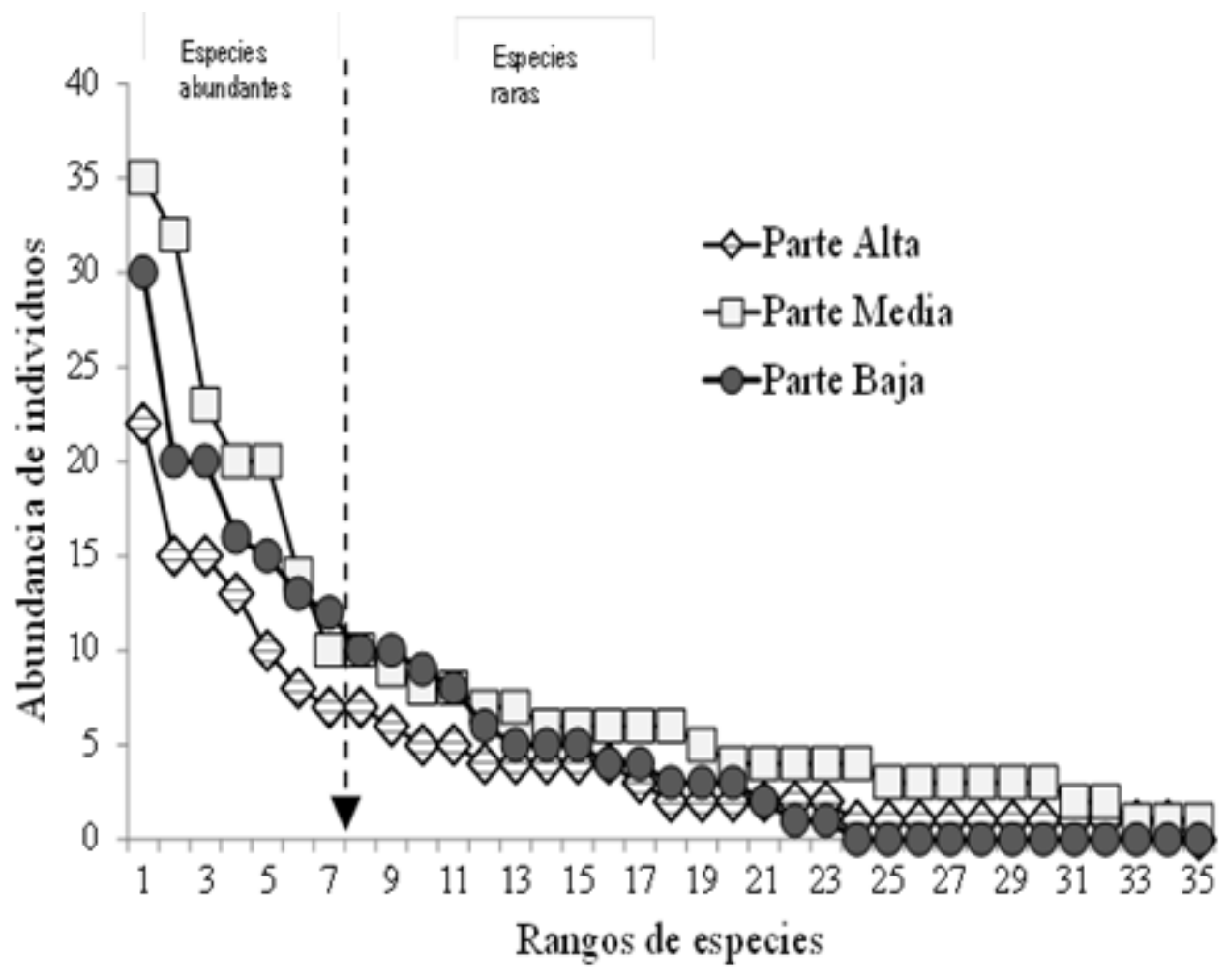

Figura 5. Rangos de abundancias de especies florísticas de las partes bajas, medias y altas de la Microcuenca de El Caraño, Quibdó, Chocó 
Bioetnia Volumen 9 № 2 (julio-diciembre), 2012

Tabla 2

Í ndices de diversidad de especies florísticas en la microcuenca de El Caraño, de Quibdó, Chocó

\begin{tabular}{lrrrr}
\hline \multicolumn{1}{c}{ Índices ecológicos } & alta & $\begin{array}{c}\text { Zona } \\
\text { media }\end{array}$ & baja & Total \\
\hline Riqueza de especies & 34 & 35 & 23 & 71 \\
Abundancia de individuos & 159 & 283 & 205 & 647 \\
Diversidad de Margalef & 6.51 & 6.023 & 4.133 & 10.82 \\
Dominancia de Simpson & 0.0623 & 0.05782 & 0.07174 & 0.0323 \\
Equidad de Shannon-Wiener & 3.084 & 3.163 & 2.837 & 3.741 \\
\hline
\end{tabular}

(Figura 5) donde se observan muchos individuos en pocas especies (menos de diez especies concentran más del 60\% de los individuos) y un número elevado de especies que están representadas por pocos individuos. El hecho de que las zonas media y baja presenten valores de equidad menor que el registrado en la zona alta, sugiere la posibilidad que estas dos áreas de la microcuenca hayan estado sometidas a procesos agresivos de colonización por parte de especies invasoras que aumentan sus densidades rápidamente desplazando así a las nativas.

Consideraciones para la rehabilitación y conservación de la microcuenca EI Caraño. En este sentido, al evaluar las especies vegetales encontradas en este trabajo desde el punto de vista de su vulnerabilidad, considerando los listados de especies amenazadas de la IUCN, no se encontró ninguna especie dentro de la categoría de amenaza. Sin embargo, en términos generales la vegetación de la microcuenca El Caraño está caracterizada por elementos representativos de áreas que han sido sometidas a disturbios fuertes y recurrentes que generan modificaciones a nivel la estructura y composición como bien se evidenció en los resultados. A pesar de esto, los altos niveles de diversidad que presenta la vegetación de la cuenca, definidos especialmente por el alto número de especies raras, sobre todo en la zona alta, donde aún se pueden apreciar remanentes de bosques estratificado, hace necesario implementar estrategias inter institucionales que permitan la puesta en marcha de medidas que lleven a una gestión sostenible de esta importante microcuenca para el pueblo quibdoseño y así evitar el deterioro total.

La microcuenca El Caraño es de mucha importancia para la ciudad de Quibdó, porque representa un gran potencial de suministros de agua y recursos de subsistencia para parte de la población quibdoseña. Sin embargo, el acelerado y desordenado proceso de crecimiento de la ciudad de Quibdó en los últimos años, ha dejado a esta importante microcuenca en una situación crítica desde el punto de vista de su preservación para las futuras generaciones desde el punto de vista de los bienes y servicios ambientales que potencialmente podría proveer a las comunidades asentadas en su área de influencia.

A continuación se listan algunas directrices para la rehabilitación de los bosques de la microcuenca El Caraño:

Políticas, empoderamiento y gobernanza. Incorporar los bosques degradados a las políticas locales de planificación del uso de tierras a nivel local y regional donde se establezcan planes integrados de uso de tierras que reflejen un equilibrio adecuado entre las necesidades de uso de la tierra, la conservación y la producción de los bosques.

Plantación de plántulas de árboles nativos. La estrategia usada más comúnmente para acelerar la sucesión de áreas degradadas es plantar plántulas o juveniles de unas pocas especies de árboles nativos de rápido crecimiento, resistentes a inundaciones y capaces de crecer en suelos con pocos nutrientes. La siembra directa puede ser una opción viable, sin embargo, hay que tener en cuenta que la invasión de malezas y las tasas de depredación son a menudo lo suficientemente altas como para imposibilitarla.

Árboles remanentes y plantación de grupos de árboles. Los árboles remanentes desempeñan un papel crítico en la recuperación de la cobertura boscosa nativa al aumentar la dispersión de semillas, mejorar las condiciones microclimáticas e incrementar los nutrientes del suelo. Debe fomentarse la no tala de árboles semilleros en la zona alta de la microcuenca para facilitar su recuperación. La importancia de los árboles aislados y de los grupos de árboles para facilitar la dispersión de semillas y el establecimiento de plántulas sugiere que la plantación de grupos de árboles puede ser un método eficaz en función de los costos para facilitar la recuperación. Este método puede también proporcionar un nivel de diversidad espacial característico del ecosistema.

Siembra de arbustos. Los arbustos que son naturalmente colonizadores pueden desempeñar un papel crítico en el mejoramiento de condiciones adversas y contribuir a la sucesión en pastizales tropicales abandonados. La siembra de arbustos de sucesión temprana puede ser una estrategia económica para acelerar la recuperación en regiones donde los arbustos facilitan el establecimiento de plántulas de 
Intervención antrópica y transformación de la vegetación. LA. Mosquera-Maturana et al.

árboles, ya que muchos arbustos, a diferencia de la mayoría de las especies arbóreas, producen todo el año cantidades abundantes de semillas que son fáciles de recolectar.

\section{Literatura citada}

Asprilla, A. R., C. M. Mosquera, H. Valoyes, H. Cuesta, F. García. 2003. Composición florística de un bosque pluvial tropical (bp-T) en la Parcela Permanente de Investigación en Biodiversidad (PPIB) en Salero, Unión Panamericana, Chocó. En: García, F, Ramos, Y. A., Palacios, J., Arroyo, J.E., Mena, A., González, M. (Eds.). Salero: Diversidad Biológica de un Bosque Pluvial tropical (bp-T). Bogotá: Guadalupe Ltda. 39-44 pp.

Bonilla-Luna, N., H. Cuesta-Córdoba. H. Valois-Cuesta H. 2011. Efectos de la extracción forestal sobre la estructura y composición de un bosque pluvial del Pacífico colombiano. Biodiversidad Neotropical. 1(1): 4854.

Díaz, W. A., S. El Coro. 2009. Plantas colonizadoras en áreas perturbadas por la minería en el estado Bolívar, Venezuela. Acta Bot Venez. 32 (2): 45366.

Forero, E., A. H. Gentry. 1989. Lista anotada de plantas del departamento del Chocó. Bogotá: Instituto de Ciencias Naturales, Universidad Nacional de Colombia, Museo de Historia Natural. 143 p.

Gentry, A. H. 1996. A field guide to the families and genera of woody plants of Northwest South America (Colombia Ecuador and Peru) con supplementary notes on herbaceous taxa. Chicago: University of Chicago Press.

Herbario COL. En línea. [fecha de acceso: 20 de mayo de 2012] disponible en: URL http://www.biovirtual.unal.edu.co.

Missouri Botanical Garden (MBG). Base de datos del trópico. En línea. [fecha de acceso: 17 de mayo de 2012] disponible en: URL: http:// www.tropicos.org
Mosquera, N., K. D. Mosquera. 2002. Comparación de la estructura y composición de bosques secundarios pluviales tropicales con diferentes grados de intervención antrópica. Trabajo de grado. Programa de Biología con Énfasis en Recursos Naturales. Quibdó: Universidad Tecnológica del Chocó. 124 p.

Palacios, J., Y. A. Ramos, A. K. Mosquera, C. Castro, F. García, J. E. Arroyo, A. Cogollo. 2003. Estructura de un bosque pluvial tropical (bp-T) en Salero, Unión Panamericana, Chocó. En: García, F, Ramos, Y. A., Palacios, J., Arroyo, J.E., Mena, A., González, M. (Eds.) Salero: Diversidad Biológica de un Bosque Pluvial tropical (bp-T). Bogotá: Guadalupe Ltda. 45-61 pp.

Poveda-M., C., C. A. Rojas-P., A. Rudas-L1., O. Rangel-Ch. 2004. El Chocó biogeográfico: Ambiente biofísico. En: Rangel-Ch. O. (ed.) Colombia diversidad biótica IV: El Chocó biogeográfico. Bogotá: Universidad Nacional de Colombia. 1-21 pp.

$\mathrm{R}$ Core Team. 2012. A language and environment for statistical computing. $\mathrm{R}$ Foundation for Statistical Computing. http://www.R-project.org/

Ramírez, A. 2006. Ecología: métodos de muestreo y análisis de poblaciones y comunidades. Bogotá: Pontificia Universidad Javeriana. 271 p.

Rangel-Ch., O., O. Rivera-Díaz. 2004. Diversidad y riqueza de espermatofitos en el Chocó biogeográfico. En: Rangel-Ch. O. (ed.) Colombia diversidad biótica IV: El Chocó biogeográfico. Bogotá: Universidad Nacional de Colombia. 83-99 pp.

Sarmiento, G., M. Pinillos, I. Garay. 2005. Biomass variability in tropical American lowland rainforest. Ecotropicos 18 (1): 1-20.

The International Plant Names Index. En línea. [fecha de acceso: 20 de mayo de 2012]. URL disponible en: http://www.ipni.org

The plant list. En línea. [fecha de acceso: 30 de mayo de 2012] URL disponible en: http://www.theplantlist.org

Villareal, H., M. Álvarez, S, Córdoba, F. et al. 2006. Manual de métodos para el desarrollo de invQentarios de biodiversidad. Bogotá: Instituto de Investigación de Recursos Biológicos Alexander von Humboldt. 236 pp. 